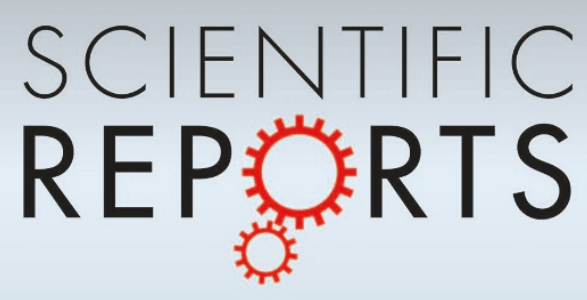

\title{
OPEN Gelling by Heating
}

SUBJECT AREAS:

COLLOIDS

SELF-ASSEMBLY

FLUIDS

COMPUTATIONAL METHODS

Received

9 May 2013

Accepted

25 July 2013

Published

16 August 2013

Correspondence and requests for materials should be addressed to S.R.V. (sandalo@ugr.

\author{
Sándalo Roldán-Vargas' ${ }^{1}$, Frank Smallenburg' ${ }^{1}$, Walter Kob² \& Francesco Sciortino'
}

'Department of Physics, Sapienza, Università di Roma, Piazzale Aldo Moro 2, I-00 185, Roma, Italy, ${ }^{2}$ Laboratoire Charles Coulomb, UMR 5221, CNRS and Université Montpellier 2, F-34095 Montpellier, France.

We exploit the concept of competing interactions to design a binary mixture of patchy particles that forms a reversible gel upon heating. Our molecular dynamics computer simulation of such a system shows that with increasing temperature the relaxation dynamics slows down by more than four orders of magnitude and then speeds up again. The system is thus a fluid both at high and at low temperatures and a solid-like disordered open network structure at intermediate temperature. We further discuss the feasibility of realizing a real material with this reversible behavior.

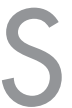

ome of the most versatile and efficient strategies for designing new materials with unconventional behavior are based on the idea of competitive interaction. While in biology the term competitive interaction indicates the result of rivalry between two or more species competing for resources, in physics it stands for the presence of several interaction mechanisms that can stabilize competing local structures, leading to novel and highly interesting features of the system. Recent examples for this mechanism in soft matter systems include, among others, the competition between short-range attraction and long-range repulsion in charged colloids giving rise to cluster phases ${ }^{1-3}$, the competition between chaining and branching in patchy colloids ${ }^{4,5}$ where a specific design of the inter-patch interactions results in a phase diagram in which the density of the coexisting liquid approaches the density of the gas ${ }^{5}$, and the design of DNA-coated colloids with two different DNA sequences for the purpose of establishing a competition between intra and inter-particle interactions, favoring crystal formation ${ }^{6}$. Often the very nature of these competing mechanisms promotes the emergence of a structure controlled by energy (stable at low temperature, $T$ ) which competes with a structure stabilized by entropy at intermediate $T$.

Recent progress in the synthesis of colloids has led to a novel type of particles that have highly directional and selective interactions, thus providing valence to colloids ${ }^{7}$. In particular the recent developments in large scale synthesis of patchy colloidal particles ${ }^{8}$ and in DNA nanotechnology have already allowed for the production of versatile constructs in which specificity, valence and interaction strength can be tuned at will ${ }^{9,10}$. This ability to control the interactions between nano-and meso-sized particles ${ }^{8}$, and to design the geometric properties of the patches $^{11,12}$ and/or their functionalization, offers now the possibility to take advantage of the competitive interactions that are usually present in such systems and hence to modulate material properties with external control parameters. In this Report we develop one such possibility: The design of a material that continuously (i.e. without the interference of a first order transition) and reversibly gels upon heating. In contrast to gelation on heating observed in protein solutions ${ }^{13}$, where kinetic arrest is driven by the irreversible denaturation of the proteins, here we require thermo-reversibility of the process and a simple design of the constituent particles. We show how a binary mixture of limited valence particles can provide a model in which the competition between entropy and potential energy causes the system to show a re-entrant behavior, passing reversibly from a fluid to a gel and again to a fluid if $T$ is varied. The proposed model, amenable to theoretical analysis and experimental realization, shows in simulations a slowing down of the dynamics of several orders of magnitude on heating, providing the first example of controlled reversible gelation.

Patchy colloidal particles with a limited number $f$ of attractive patches progressively cluster when cooled, leading such systems to form a percolating network that at sufficiently low $T$ will contain all particles in the system $^{14-19}$. Due to this aggregation, the dynamics slows down by several orders of magnitude. In the network state the lifetime of the bonds (a $T$-controlled quantity) fixes the timescale over which the system behaves as a solid. It is known that if $f=4$, the particles (here called $A$ species) form a random tetrahedral network which closely resembles the structure of network-forming atomic systems like silica or silicon ${ }^{20}$. To melt the network at low $T$, we add a second species $(B)$ with only a single patch which competes for bonding with the patches on the network forming $A$-species ${ }^{21}$. The idea is to design a competitive mechanism such that the bonding between $A$ - and $B$ particles becomes dominant, but only at a temperature which is much lower than the one at which the $A A$ network is formed. As a result, the stable low- $T$ phase consists of $A$-particles decorated with $f B$-particles which are 
free to diffuse in the sample volume, whereas at intermediate $T$ the system forms a highly viscous $A A$-network that is progressively fragmented and transformed into a fluid upon heating.

\section{Results}

Model. We consider a binary mixture of patchy colloids where each $A$-particle has $f=4$ patches on its surface that are arranged in a tetrahedral geometry and the $B$-particles have only one patch (see Fig. 1). The patch-patch interaction is modeled via a Kern-Frenkel potential $^{22}$, a model that has been extensively used over the last decade to compare simulations and experiments on the selfassembly of patchy colloids ${ }^{23-25}$. Each $A$-patch can interact either with a $B$-patch with unit energy $\epsilon_{A B}$ and bonding volume $\mathcal{V}_{A B}$ or with another $A$-patch with energy $\epsilon_{A A}=0.95 \epsilon_{A B}$ and $\mathcal{V}_{A A}\left(\gg \mathcal{V}_{A B}\right)$. No $B B$-bonding is allowed. The bonding volumes are each determined by an interaction range $\delta_{\alpha}$ and an angular patch width $\theta_{\alpha}(\alpha \in\{\mathrm{AA}, \mathrm{AB}\})$ (see Fig. 1 and Methods). The attractive patchpatch interaction is complemented by an isotropic hard-core repulsion, where the spherical cores have diameters $\sigma_{A}$ and $\sigma_{B}=$ $0.35 \sigma_{A}$. The size ratio was chosen such that the $B$-particles can block the $A$-patches from bonding to other $A$-patches without significantly increasing the packing fraction of the pure $A$ system. Due to geometric constraints, each patch can be involved in only a single bond.

Using event-driven molecular dynamics simulations ${ }^{26-28}$ (see Methods), we have studied a system of $N_{A}=600$ and $N_{B}=2400$ particles, corresponding to a total number density $\rho \sigma_{A}^{3}=3.0$, with partial number densities $\rho_{A} \sigma_{A}^{3}=0.6$ and $\rho_{B} \sigma_{A}^{3}=2.4$, for a wide range of $T$, whose unit is given by $\epsilon_{A B} / k_{B}$, where $k_{B}$ is Boltzmann's constant. The composition of the system is thus fixed at $x_{A}=0.2$. The density $\rho_{A} \sigma_{A}^{3}=0.6$ of $A$-particles corresponds to the optimal density at which tetrahedral particles form an unstrained fully bonded network $^{29}$. With this composition, the fully bonded network has an energy of $2 N_{A} \epsilon_{A A}$, whereas a configuration in which all the $B$-particles are bonded to the $A$-particles has the significantly lower energy of $4 N_{A} \epsilon_{A B}$. We also simulate for a low $T\left(=0.04 \epsilon_{A B} / k_{B}\right)$ a reference system composed of 600 flowers (see Fig. 1d), i.e. $A$-particles bonded a)

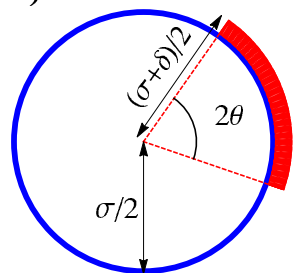

c)

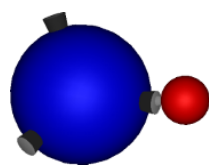

b)

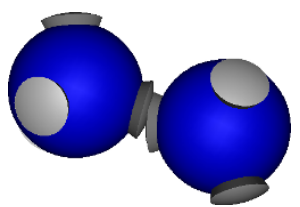

d)

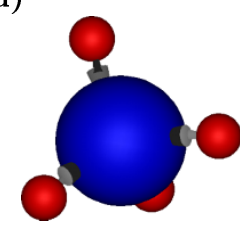

Figure $1 \mid$ The Kern-Frenkel model. (a) Schematic of the interaction parameters in the Kern-Frenkel model. An A-patch can bond with either another $A$-particle (b), or with a $B$-particle (c). Panel (d) shows a "flower", i.e. a fully bonded cluster consisting of one $A$-particle and four $B$-particles, representing the lowest-energy state of the system. Here the interaction ranges and the angular patch widths are $\cos \theta_{A A}=0.92, \delta_{A A}=0.15 \sigma_{A}$, and $\cos \theta_{A B}=0.99, \delta_{A B}=0.2 \sigma_{A}$. With these choices the bonding volumes are given by $\mathcal{V}_{A A}=3.49 \cdot 10^{-3} \sigma_{A}^{3}$ and $\mathcal{V}_{A B}=3.79 \cdot 10^{-5} \sigma_{A}^{3}$ (see Methods). Note that in the binary Kern-Frenkel model, $\theta$ and $\delta$ of each bond are defined by the species of both bonding partners and are not properties of individual particles. to four $B$-particles. At this low $T$ no bond breaking events take place within the simulation time.

Temperature dependence of the bonding probability. Figure 2 demonstrates the effect of the competitive interactions present in our system. It shows the $T$-dependence of the probability that a patch on an $A$-particle is bonded to another $A$-patch $\left(p_{\mathrm{AA}}\right)$ or to a $B$-patch $\left(p_{\mathrm{AB}}\right)$. On cooling, $p_{\mathrm{AA}}$ starts to grow, signaling the onset of the network formation, and reaches a maximum around $T=$ $0.11 \epsilon_{A B} / k_{B}$. We recall that within a mean-field description, percolation of particles with valence $f=4$ takes place at $p_{\mathrm{AA}}=1 / 3^{30}$. Since at the maximum we find $p_{\mathrm{AA}} \approx 0.9$ we can conclude that at this $T$ the $A$-particles have formed a highly bonded percolating network. Upon further cooling, $p_{\mathrm{AA}}$ starts to decrease whereas $p_{\mathrm{AB}}$ grows quickly, showing that the $A B$-bonds are starting to replace the $A A$-bonds. The entropy associated with the larger bonding volume $\mathcal{V}_{A A}$ for the $A A$ interaction is crucial for promoting the formation of a large number of $A A$-bonds at intermediate $T$, before the energetically preferred but entropically disfavored $A B$-bonds set in. Figure 2 also shows the parameter-free theoretical predictions for the bonding probabilities as obtained from the first-order thermodynamic perturbation theory developed by Wertheim ${ }^{31-33}$ (details on the Wertheim calculations are reported in the Methods section). The Wertheim theory nicely captures the mechanism of competing interactions, reproducing the position and height of the maximum of $p_{\mathrm{AA}}$ as well as the low $T$ trends of $p_{\mathrm{AA}}$ and $p_{\mathrm{AB}}$.

Structure. Figure 3 shows the unusual $T$ dependence of the structure of the system, related to the non-monotonic behavior of $p_{A A}$. At high $T$, the partial structure factor $S_{A A}(q)$ shows the conventional $q^{-}$ dependence found in simple liquids with a main peak around $q \sigma_{A}$ $=7.2$. Upon decreasing $T$ the main peak splits into two, one located around $q \sigma_{A}=5.2$ and a higher one around 8.4. This double peak feature is typical of liquids that have a local tetrahedral network structure, such as silicon or silica ${ }^{20}$. The peak at $q \sigma_{A} \approx 8.4$ corresponds to the nearest neighbor distance between two bonded $A$-particles, whereas the one at around 5.2 is associated with the second-nearest neighbors in the tetrahedral network. Note that this double peak structure is most pronounced at $T \approx 0.11 \epsilon_{A B} / k_{B}$, i.e. at the $T$ at which $p_{A A}$ has a maximum (see Fig. 2) and hence the gel is maximally connected. When $T$ is lowered even further the double peak structure disappears and $S_{A A}(q)$ becomes again similar to the

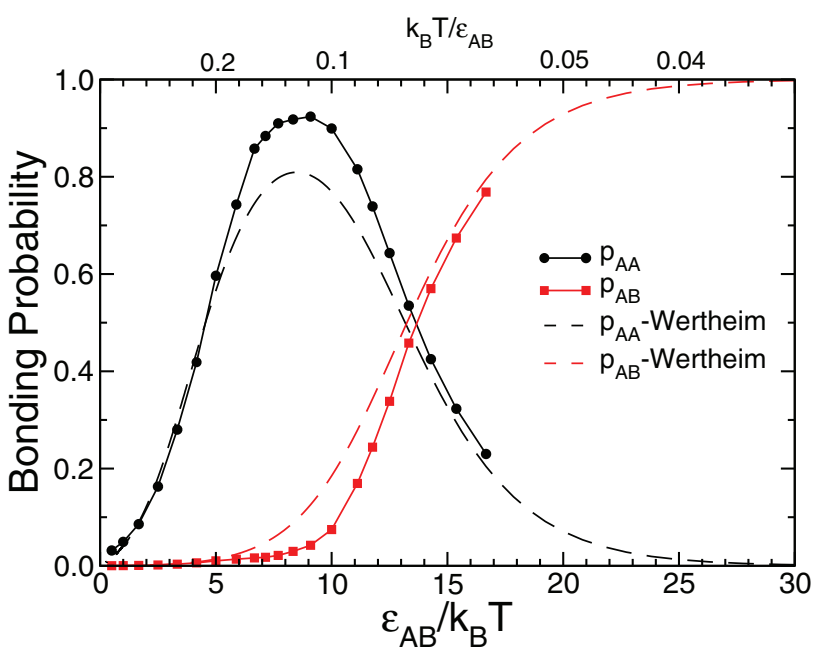

Figure $2 \mid$ Bonding probabilities. Probability that a patch on an $A$-particle is bonded to another patch on an $A$-particle, $p_{\mathrm{AA}}$ (black circles), or to a patch on a $B$-particle, $p_{\mathrm{AB}}$ (red squares). The dashed lines are the prediction for these probabilities as calculated from the Wertheim theory. 


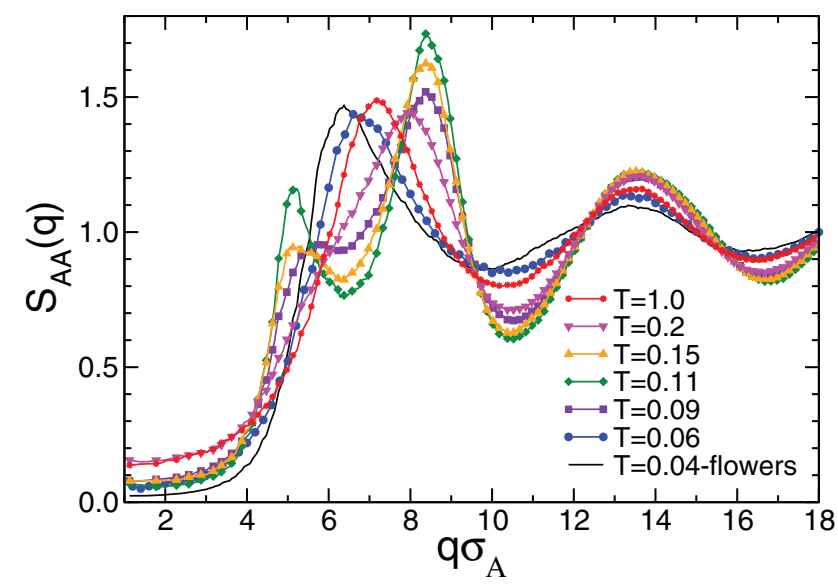

Figure $3 \mid$ Structure factor. Partial structure factor $S_{\mathrm{AA}}(q)$ for different values of $T$ (solid lines with different symbols). The $S_{\mathrm{AA}}(q)$ for a fluid of flowers at $T=0.04 \epsilon_{A B} / k_{B}$ (black solid line) is also represented.

structure factor of a fluid composed of flowers (which is also included in Fig. 3 as a reference). Since the size of a flower is larger than that of an $A$-particle, the peak position at low $T$ is to the left of the one observed at high $T$.

Temperature dependence of the diffusion coefficient. We now quantify the effect of the competing interactions on the dynamics of the system and provide evidence that the change of the structure rich in $A B$-bonds at low $T$ to the highly bonded $A A$-network generates a slowing down of the dynamics on heating. For this, we calculate the mean squared displacement (MSD) for the particles of both species and then their corresponding diffusion coefficients $D_{\alpha}$ $(\alpha \in\{A, B\})$ from the long-time behavior of the MSD via the Einstein relation. We should note that the center of mass (CM) of a single species has a non-zero velocity (which is compensated by the CM motion of the other species). To obtain meaningful results for the MSD of a single species, we subtract the CM drift of the species in question before evaluating the MSD. In addition, to remove the trivial trend originated from the $T$-dependence of the thermal velocity we divide $D_{\alpha}$ by a reference diffusion coefficient $D_{0} \equiv \sigma_{A}^{2} /$ $\tau_{0}$, where $\tau_{0}=\sqrt{m_{A} \sigma_{A}^{2} / k_{B} T}$ and $m_{A}$ is the mass of an $A$-particle.

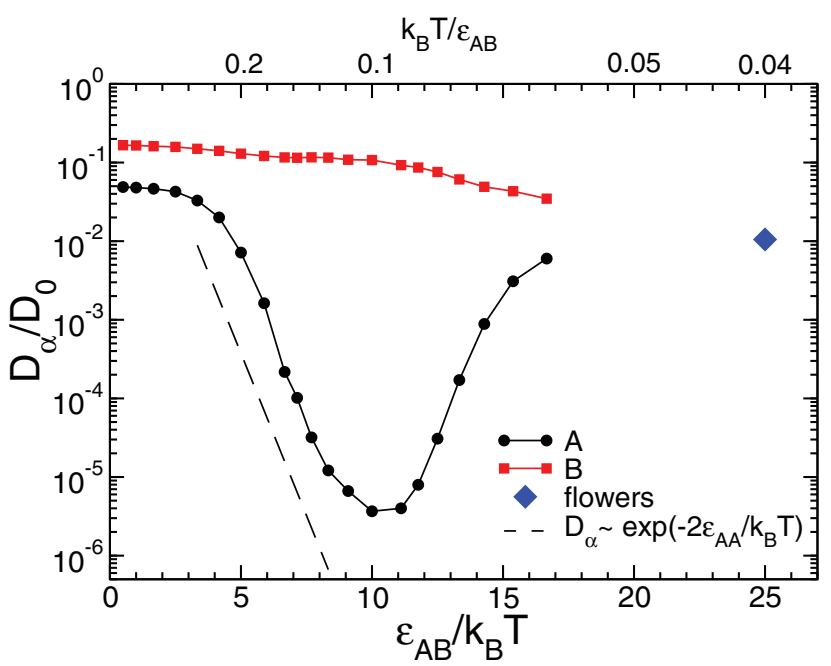

Figure $4 \mid$ Diffusion coefficient. Arrhenius plot of the normalized diffusion coefficient $D_{\alpha} / D_{0}(\alpha=A$, circles, $\alpha=B$, squares $)$. Also included is an Arrhenius law with activation energy $2 \epsilon_{A A}$ (dashed line). The diffusion coefficient of a fluid of flowers at low $T$ is represented by a blue diamond.
Figure 4 shows the $T$-dependence of $D_{\alpha}$ in an Arrhenius plot. At high $T, D_{\alpha}$ is approximately constant for both type of particles, indicating that bonds do not play a significant role. On cooling, $D_{A}$ starts to decrease very rapidly, with a super-Arrhenius $T$-dependence reminiscent of that observed in molecular networks ${ }^{20}$, turning into an Arrhenius law with an activation energy approximately equal to $2 \epsilon_{A A}$ (see dashed line in Fig. 4). Similar values of the activation energy are typically found in tetrahedral network-forming systems where most of the particles belong to the percolating cluster, and bond breaking is the bottleneck for relaxation ${ }^{18,34,35}$. Before the gel starts to decompose at a temperature below $T \approx 0.11 \epsilon_{A B} / k_{B}, D_{A}$ has already decreased by four orders of magnitude compared to its value at high $T$, indicating the formation of a persistent network. For $T \lesssim 0.11 \epsilon_{A B} / k_{B}, D_{A}$ starts to increase. This rising persists down to the lowest $T$ at which we were able to equilibrate the system. We emphasize that this non-monotonic $T$-dependence is only observed for the $A$-particles, i.e. the particles which are involved in the formation of the network. In contrast, $D_{B}$ shows only a rather mild $T$-dependence. Fig. 4 also shows the diffusion coefficient of the fluid of flowers at $T=0.04 \epsilon_{A B} / k_{B}$ for which $D_{A}=D_{B}$, the common value to which both $D_{A}$ and $D_{B}$ converge.

Relaxation times. A non-monotonic behavior of the characteristic time is also found in the time evolution of the collective- and selfintermediate scattering functions. Their study provides insight into how the relaxation dynamics depends on the considered length scale. Figure 5a shows an Arrhenius plot of the relaxation time $\tau_{A}(q)$

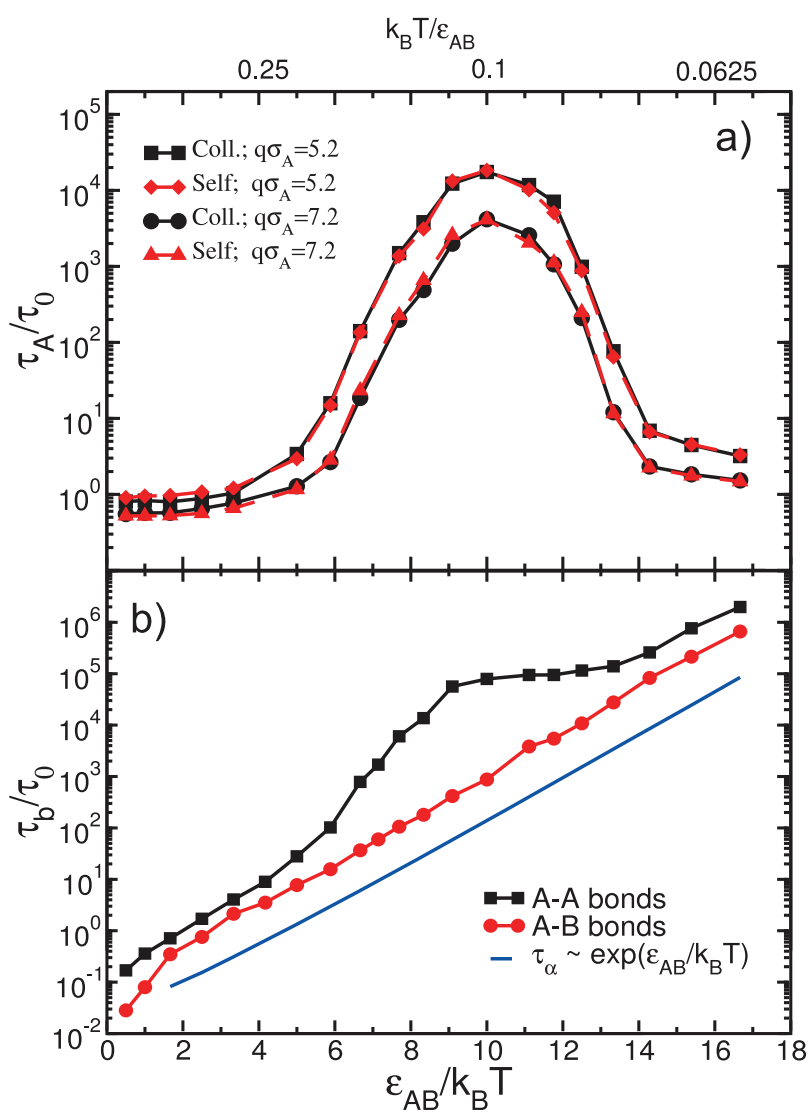

Figure $5 \mid$ Relaxation times. (a) Arrhenius plot of the normalized relaxation time $\tau_{A}(q) / \tau_{0}$ as obtained from the self (dashed lines) and collective (solid lines) scattering functions, where $\tau_{0}=\sqrt{m_{A} \sigma_{A}^{2} / k_{B} T}$. The different curves correspond to the wave-vectors given by the first two peaks in $S_{A A}(q)$. (b) Arrhenius plot of the normalized bond-persistence time $\tau_{b} /$ $\tau_{0}$ for the $A A$ (squares) and $A B$ bonds (circles). Also included is an Arrhenius law with activation energy $\epsilon_{\mathrm{AB}}$ (blue solid line). 
determined from the time integral of the intermediate scattering function of the $A$-particles for two different $q$-vectors: $q \sigma_{A}=5.2$ and $q \sigma_{A}=7.2$, which correspond, respectively, to the location of the first peak in the network and in the high- $T$ fluid (see Fig. 3 ). We find that the self and collective relaxation times $\tau_{A}(q)$, normalized by $\tau_{0}$, show qualitatively the same $T$-dependence: a plateau at high $T$, a fast increase within the $T$-range in which the network is formed, a quick decrease once the network starts to break up again, and a final plateau at low $T$. This $T$-dependence is observed for both values of $q$, indicating that the relaxation mechanism does not depend on the length scale considered.

To provide further evidence that the system is ergodic on long time scales, i.e. that the structure of the system has completely lost its memory of the initial state, we have investigated the bond persistence function $p_{b}(t)$, i.e. the probability that a bond which is present at time zero is also present at time $t$ (see Supplementary Information). Hence, the relaxation time of $p_{b}(t)$ provides information on the restructuring time of the network connectivity. Figure $5 b$ shows an Arrhenius plot with the $T$-dependence of the decay time $\tau_{b}$, where $p_{b}\left(\tau_{b}\right)=e^{-1}$. At intermediate and low $T, \tau_{b}$ is larger than the relaxation times shown in Fig. $5 \mathrm{a}$. We find thus that $p_{b}(t)$ decays to zero only on a time scale that is significantly longer than the relaxation times associated with the scattering functions, confirming that some fraction of spacial decorrelation of the network, as quantified by the collective scattering function, takes place at partially fixed bonding pattern. The extreme case occurs at very low $T$ where the system is a fluid of flowers that relaxes relatively quickly but in which $A B$-bonds survive for a very long time. Figure $5 \mathrm{~b}$ also shows that the $T$-dependence of the lifetime of the $A A$-bonds differs strongly from the one of the $A B$-bonds. The latter shows in the whole $T$-range an Arrhenius dependence with an activation energy very close to $\epsilon_{\mathrm{AB}}$ (see blue solid line in Fig. 5b). This suggests that the mechanism for the breaking of an $A B$-bond is not collective in nature but a simple activated process. In contrast, the breaking time for an $A A$-bond follows an Arrhenius law at high $T$ but becomes super-Arrhenius within the $T$-region in which the gel forms, showing that this bond-breaking process is of collective nature. At very low $T$ the effective activation energy becomes again an Arrhenius behavior with an activation energy given by $\epsilon_{\mathrm{AA}}$.

\section{Discussion}

We have demonstrated that a judicious choice of the interaction parameters of a binary mixture of $A$ and $B$ patchy particles shows a non-monotonic and reversible $T$-dependence of its dynamic properties. We set up a competition between network-forming $A A$-bonds and network-breaking $A B$-bonds, which are favored by entropy and energy, respectively. Thus, at intermediate $T$, entropy stabilizes a viscous network gel, whereas both at low and high $T$, the network breaks up, leading to a simple fluid state. Our results are robust with respect to a change in the model parameters and we have indeed observed a qualitatively similar phenomenology in our system for a wide range of the interaction energy and density, $0.85 \lesssim \epsilon_{A A} / \epsilon_{A B}$ $\lesssim 1.0$ and $2.5 \lesssim \rho \sigma_{A}^{3} \lesssim 3.5$. Since our system requires no fine-tuning, we expect that the observed phenomenon can also be realized experimentally. In particular, two experimental systems are very promising candidates to test the ideas presented in this Report: (i) a solution of DNA constructs of valence four ${ }^{36,37}$ in the presence of competing DNA single strands and (ii) the binary mixture of patchy particles recently synthesized in Ref. 8. Both of these systems offer singlepatch and four-patch colloidal particles with controllable interaction strengths that are already amenable for experimentation, and thus have the potential to provide soft materials that reversibly gel upon heating.

\section{Methods}

Kern-Frenkel model. The particles investigated in the paper are modeled via the wellknown Kern-Frenkel model ${ }^{22}$. In this model, particles interact by means of a combination of a hard-sphere potential $u_{\mathrm{HS}}$ and an attractive directional interaction $u_{\text {patch. }}$ The hard-core repulsion between two particles $i$ and $j$ is given by:

$$
\beta u_{\mathrm{HS}}\left(r_{i j}\right)=\left\{\begin{array}{ll}
\infty & \text { if } r_{i j}<\sigma_{i j} \\
0 & \text { otherwise }
\end{array},\right.
$$

here, $\beta=1 / k_{B} T$, with $k_{B}$ Boltzmann's constant and $T$ the temperature, $r_{i j}$ is the center to-center distance between the particles, and $\sigma_{i j}=\left(\sigma_{i}+\sigma_{j}\right) / 2$ denotes the contact distance between the particles, with $\sigma_{i}\left(\sigma_{j}\right)$ the diameter of particle $i(j)$. The sitespecific attraction between the particles is determined by the circular patches on the surface of each particle, which interact such that two particles form a bond with interaction energy $\epsilon_{i j}$ when i) their centers of mass are within a maximum interaction range $\sigma_{i j}+\delta_{i j}$, and ii) the center-to-center vector between the particles passes through a patch on the surface of both particles (see Fig. 1 of main text). The size of the patches is determined by an opening angle $\theta_{i j}$. The potential energy of two particles $i$ and $j$ is thus given by

$$
u_{\text {patch }}\left(\mathbf{r}_{i j},\left\{\mathbf{p}_{i}\right\}\left\{\mathbf{p}_{j}\right\}\right)=u_{\mathrm{SW}}\left(r_{i j}\right) \Phi\left(\mathbf{r}_{i j},\left\{\mathbf{p}_{i}\right\}\right) \Phi\left(\mathbf{r}_{j i},\left\{\mathbf{p}_{j}\right\}\right),
$$

where $u_{\mathrm{SW}}$ is a square-well attraction, given by:

$$
u_{\mathrm{SW}}\left(r_{i j}\right)= \begin{cases}-\epsilon_{i j} & \text { if } r_{i j}<\sigma_{i j}+\delta_{i j} \\ 0 & \text { otherwise }\end{cases}
$$

The function $\Phi\left(\mathbf{r}_{i j},\left\{\mathbf{p}_{i}\right\}\right)$ is defined as

$$
\Phi\left(\mathbf{r}_{i j},\left\{\mathbf{p}_{i}\right\}\right)= \begin{cases}1 & \text { if } \hat{\mathbf{r}}_{i j} \cdot \mathbf{p}>\cos \left(\theta_{i j}\right) \text { for any } \mathbf{p} \text { in }\left\{\mathbf{p}_{i}\right\} \\ 0 & \text { otherwise }\end{cases}
$$

where $\mathbf{r}_{i j}=\mathbf{r}_{j}-\mathbf{r}_{i},\left\{\mathbf{p}_{i}\right\}$ is a set of normalized vectors pointing from the center of particle $i$ towards the center of each of its patches, and $\hat{\mathbf{r}}$ is a unit vector in the direction of $\mathbf{r}$.

In the model used in the Report, all particles are either species $A$ or species $B$. The $A$-particles (with hard sphere diameter $\sigma_{A}$ ) have four patches each, arranged in a tetrahedral geometry. The $B$-particles are smaller, with $\sigma_{B}=0.35 \sigma_{A}$, and have only a single patch. The parameters for the interaction between two $A$-particles are given by $\cos \theta_{A A}=0.92$, and $\delta_{A A}=0.15 \sigma_{A}$. For the $A B$-interactions, $\cos \theta_{A B}=0.99$, and $\delta_{A B}=$ $0.2 \sigma_{A}$. There are no attractive interactions between the $B$-particles. The ratio between the two interaction strengths is fixed at $\epsilon_{A A}=0.95 \epsilon_{A B}$.

Event-driven molecular dynamics. To study the relaxation dynamics of our model we use event-driven Molecular Dynamics (EDMD) simulations ${ }^{26,27}$. The implementation of the EDMD simulation relies on the numerical prediction of bond formation and bond breaking events, and follows the same scheme as described in Ref. 28. In the model under consideration here, the mass $m$ of each particle is taken to be the same, setting the time unit of the simulation $\tau_{0}=\sqrt{\beta m \sigma_{A}^{2}}$. Similarly, the moments of inertia tensors of all particles were also chosen to be the same: $I_{x x}=I_{y y}=I_{z z}=m \sigma_{A}^{2}$.

During the equilibration of the simulations, the temperature is controlled by an Andersen thermostat: periodically, randomly selected particles are given a new velocity and angular velocity, drawn from a Maxwell-Boltzmann distribution. While measuring the diffusion coefficient, no thermostat is used, so that the total energy in the system is constant. To speed up equilibration of the system at low temperature, the initial configuration is taken from a standard canonical Monte Carlo simulation equilibrated at the same temperature $T$.

Wertheim theory. Wertheim's thermodynamic perturbation theory allows us to obtain an analytical expression for the free energy of pure fluids and fluid mixtures (a detailed description can be found in Refs. 31, 32). In the context of Wertheim's theory, and specialized to our system, the probability $p_{\alpha}$ that an $\alpha$-site $(\alpha \in\{A, B\})$ is bonded is obtained through the law of mass action, which in our case takes the form of a set of two coupled equations ${ }^{33}$ :

$$
\begin{gathered}
p_{A}=1-\left[1+\rho \sigma_{A}^{3}\left[4 x_{A}\left(1-p_{A}\right) \Delta_{A A}+\left(1-x_{A}\right)\left(1-p_{B}\right) \Delta_{A B}\right]\right]^{-1} \\
p_{B}=1-\left[1+\rho \sigma_{A}^{3}\left[4 x_{A}\left(1-p_{A}\right) \Delta_{A B}\right]\right]^{-1},
\end{gathered}
$$

where $x_{A}=0.2$ is the molar fraction of the species $A, \rho$ is the total number density. All interaction parameters needed for describing bonding between $A A$-and $A B$ enter in $\Delta_{A A}$ and $\Delta_{A B}$

$$
\begin{aligned}
\Delta_{A A} & =g_{A A}\left(\sigma_{A}\right)\left[\exp \left(\epsilon_{A A} / k_{B} T\right)-1\right] \mathcal{V}_{A A} / \sigma_{A}^{3} \\
\Delta_{A B} & =g_{A B}\left(\sigma_{A B}\right)\left[\exp \left(\epsilon_{A B} / k_{B} T\right)-1\right] \mathcal{V}_{A B} / \sigma_{A}^{3},
\end{aligned}
$$

where $\epsilon_{A A}$ and $\epsilon_{A B}$ are the bonding interaction energies of the $A A$ - and $A B$-bonds. In the present work we have approximated $\Delta_{A A}$ and $\Delta_{A B}$ by using the contact values of the partial radial distribution functions ${ }^{33}, g_{A A}\left(\sigma_{A}\right), g_{B B}\left(\sigma_{B}\right)$, and $g_{A B}\left(\sigma_{A B}\right)$ (where $\sigma_{A B}$ $\left.=\left(\sigma_{A}+\sigma_{B}\right) / 2\right)$, for a binary mixture of hard spheres as obtained from the PercusYevick Equation ${ }^{38}$ : 


$$
\begin{gathered}
g_{\alpha \alpha}\left(\sigma_{\alpha}\right)=\left\{(1-\xi)+\frac{3}{2} \sigma_{\alpha} X\right\}(1-\xi)^{-2}, \alpha \in\{A, B\} \\
g_{A B}\left(\sigma_{A B}\right)=\left[\sigma_{B} g_{A A}\left(\sigma_{A}\right)+\sigma_{A} g_{B B}\left(\sigma_{B}\right)\right] / 2 \sigma_{A B},
\end{gathered}
$$

where $\xi=\frac{\pi}{6}\left(\rho_{A} \sigma_{A}^{3}+\rho_{B} \sigma_{B}^{3}\right), X=\frac{\pi}{6}\left(\rho_{A} \sigma_{A}^{2}+\rho_{B} \sigma_{B}^{2}\right)$, being $\rho_{A}$ and $\rho_{B}$ the partial number densities of the different species. The bonding volumes $\mathcal{V}_{A A}$ and $\mathcal{V}_{A B}$ present in Equations (7) and (8) are given by:

$$
\begin{gathered}
\mathcal{V}_{A A}=\frac{4 \pi}{3}\left(\frac{1-\cos \theta_{A A}}{2}\right)^{2}\left[\left(\sigma_{A}+\delta_{A A}\right)^{3}-\sigma_{A}^{3}\right] \\
\mathcal{V}_{A B}=\frac{4 \pi}{3}\left(\frac{1-\cos \theta_{A B}}{2}\right)^{2}\left[\left(\sigma_{A B}+\delta_{A B}\right)^{3}-\sigma_{A B}^{3}\right],
\end{gathered}
$$

where $\delta_{\gamma}$ and $\theta_{\gamma}(\gamma \in\{A A, A B\})$ are respectively the interaction ranges and the angular patch widths defined in the model section. Once Eqs. (5) and (6) are solved (by using Eqs. (7)-(12)), the probabilities $p_{\mathrm{AA}}$ and $p_{\mathrm{AB}}$ that an $A$-site is specifically bonded to another $A$ - or to a $B$-site are obtained by the relations: $p_{\mathrm{AA}}=p_{A}-p_{B}$ and $p_{\mathrm{AB}}=p_{B}$.

1. Sear, R. P. \& Gelbart, W. M. Microphase separation versus the vapor-liquid transition in systems of spherical particles. J. Chem. Phys. 110, 4582-4588 (1999).

2. Groenewold, J. \& Kegel, W. K. Anomalously large equilibrium clusters of colloids. J. Phys. Chem. B 105, 11702-11709 (2001).

3. Sciortino, F., Mossa, S., Zaccarelli, E. \& Tartaglia, P. Equilibrium cluster phases and low-density arrested disordered states: The role of short-range attraction and long-range repulsion. Phys. Rev. Lett. 93, 055701 (2004).

4. Tlusty, T. \& Safran, S. A. Defect-induced phase separation in dipolar fluids. Science 290, 1328-1331 (2000).

5. Russo, J., Tavares, J. M., Teixeira, P. I. C., Telo da Gama, M. M. \& Sciortino, F. Reentrant phase diagram of network fluids. Phys. Rev. Lett. 106, 085703 (2011).

6. Angioletti-Uberti, S., Mognetti, B. M. \& Frenkel, D. Re-entrant melting as a design principle for DNA-coated colloids. Nature Mater. 11, 518-522 (2012).

7. Jones, M. R. \& Mirkin, C. A. Materials science: Self-assembly gets new direction. Nature 491, 42-43 (2012).

8. Wang, Y. et al. Colloids with valence and specific directional bonding. Nature 491, 51-55 (2012).

9. Seeman, N. C. DNA in a material world. Nature 421, 427-430 (2003).

10. Condon, A. Designed DNA molecules: principles and applications of molecular nanotechnology. Nature Rev. Genet. 7, 565-575 (2006).

11. Pawar, A. B. \& Kretzschmar, I. Patchy particles by glancing angle deposition. Langmuir 24, 355-358 (2008).

12. Romano, F. \& Sciortino, F. Patterning symmetry in the rational design of colloidal crystals. Nat. Commun. 3, 975 (2012).

13. Totosaus, A., Montejano, J. G., Salazar, J. A. \& Guerrero, I. A review of physical and chemical protein-gel induction. Int. J. Food Sci. Tech. 37, 589-601 (2002).

14. Bianchi, E., Largo, J., Tartaglia, P., Zaccarelli, E. \& Sciortino, F. Phase diagram of patchy colloids: Towards empty liquids. Phys. Rev. Lett. 97, 168301 (2006).

15. Bianchi, E., Tartaglia, P., La Nave, E. \& Sciortino, F. Fully solvable equilibrium selfassembly process: Fine-tuning the clusters size and the connectivity in patchy particle systems. J. Phys. Chem. B 111, 11765-11769 (2007).

16. Rovigatti, L. \& Sciortino, F. Self and collective correlation functions in a gel of tetrahedral patchy particles. Mol. Phys. 109, 2889-2896 (2011).

17. Russo, J., Tartaglia, P. \& Sciortino, F. Reversible gels of patchy particles: Role of the valence. J. Chem. Phys. 131, 014504 (2009).

18. Saw, S., Ellegaard, N. L., Kob, W. \& Sastry, S. Structural relaxation of a gel modeled by three body interactions. Phys. Rev. Lett. 103, 248305 (2009).

19. Saw, S., Ellegaard, N. L., Kob, W. \& Sastry, S. Computer simulation study of the phase behavior and structural relaxation in a gel-former modeled by three-body interactions. J. Chem. Phys. 134, 164506 (2011).

20. Binder, K. \& Kob, W. Glassy materials and disordered solids (World Scientific, Singapore, 2011).
21. Barcenas, M., Douda, J. \& Duda, Y. Temperature dependence of the colloidal agglomeration inhibition: Computer simulation study. J. Chem. Phys. 127, 114706 (2007).

22. Kern, N. \& Frenkel, D. Fluid-fluid coexistence in colloidal systems with shortranged strongly directional attraction. J. Chem. Phys. 118, 9882-9889 (2003).

23. Mao, X., Chen, Q. \& Granick, S. Entropy favours open colloidal lattices. Nature Mater. 12, 217222 (2013)

24. Romano, F. \& Sciortino, F. Two dimensional assembly of triblock janus particles into crystal phases in the two bond per patch limit. Soft Matter 7, 5799-5804 (2011).

25. Giacometti, A., Romano, F. \& Sciortino, F. in Janus Particles Synthesis, SelfAssembly, and Applications, edited by Granick, S. \& Jiang, S. (RCS Publishing, London, 2012).

26. Rapaport, D. C. The event-driven approach to n-body simulation. Progress of Theoretical Physics Supplement 178, 5 (2009).

27. de la Peña, L. H., van Zon, R., Schofield, J. \& Opps, S. B. Discontinuous molecular dynamics for semiflexible and rigid bodies. J. Chem. Phys 126, 074105 (2007).

28. Smallenburg, F. \& Sciortino, F. Liquids more stable than crystals. Nature Physics; doi: 10.1038/NPHYS2693 (2013).

29. De Michele, C., Gabrielli, S., Tartaglia, P. \& Sciortino, F. Dynamics in the presence of attractive patchy interactions. J. Phys. Chem. B 110, 8064-8079 (2006).

30. Flory, P. J. Principles of Polymer Chemistry (Cornell University Press, 1953).

31. Wertheim, M. S. Fluids with highly directional attractive forces. i. statistical thermodynamics. J. Stat. Phys. 35, 19 (1984).

32. Wertheim, M. S. Fluids with highly directional attractive forces. ii. thermodynamic perturbation theory and integral equations. J. Stat. Phys. 35, 35-47 (1984)

33. de las Heras, D., Tavares, J. M. \& Telo da Gama, M. M. Bicontinuous and mixed gels in binary mixtures of patchy colloidal particles. Soft Matter 8, 1785-1794 (2012).

34. Del Gado, E. \& Kob, W. Structure and relaxation dynamics of a colloidal gel. Europhys. Lett. 72, 1032-1038 (2005).

35. Del Gado, E. \& Kob, W. Length-scale-dependent relaxation in colloidal gels. Phys. Rev. Lett. 98, 028303 (2007).

36. Stewart, K. M. \& McLaughlin, L. W. Four-arm oligonucleotide ni(ii)cyclamcentered complexes as precursors for the generation of supramolecular periodic assemblies. J. Am. Chem. Soc. 126, 2050-2057 (2004).

37. Biffi, S. et al. Phase behaviour and critical activated dynamics of limited-valence DNA nano-stars. Proceeding National Academy of Science, in press.

38. Lebowitz, J. L. \& Rowlinson, J. S. Thermodynamic properties of mixtures of hard spheres. J. Chem. Phys. 41, 133-138 (1964).

\section{Acknowledgements}

We acknowledge support from COMPLOIDS and ERC-PATCHYCOLLOIDS-226207. W. Kob acknowledges support from the Institut Universitaire de France. F. Sciortino thanks F. Starr for discussions.

\section{Author contributions}

F. Smallenburg and F. Sciortino wrote the code for the EDMD and MC simulations. S. R.-V. and W. K. performed the simulations and the analysis of the data. All the authors contributed to the interpretation of the results and the writing of the paper.

\section{Additional information}

Supplementary information accompanies this paper at http://www.nature.com/ scientificreports

Competing financial interests: The authors declare no competing financial interests.

How to cite this article: Roldán-Vargas, S., Smallenburg, F., Kob, W. \& Sciortino, F. Gelling by Heating. Sci. Rep. 3, 2451; DOI:10.1038/srep02451 (2013).

(c) (i) $\Theta$ This work is licensed under a Creative Commons Attributionvisit http://creativecommons.org/licenses/by-nc-nd/3.0 